Original Contribution

\title{
GROWTH ASSESSMENT OF CYANOBACTERIA ANABAENA SP. FS 76 AND NOSTOC SP. FS 77 AFFECTED BY THERMAL SHOCK CONDITION
}

\author{
R. Taheri $^{1 *}$, S. Shokravi ${ }^{1}$, M. Hossaianzadeh ${ }^{2}$ \\ ${ }^{1}$ Department of Biology, Gorgan Branch, Islamic Azad University, Gorgan, Iran \\ ${ }^{2}$ Department of Horticultural Science, Shirvan Branch, Islamic Azad University, Shirvan, Iran
}

\begin{abstract}
Soil cyanobacteria Nostoc sp. FS 77 and Anabaena sp. FS 76 from the point of view to extreme constraints associated with light, carbon dioxide and cold shock, were evaluated. Samples were Prepared from algal museum of shahid Beheshti University and placed in vitro treatment limitations extreme light $(2$ micromoles quantum per square meter per second), carbon dioxide (non-inoculated carbon dioxide, non- aerated) temperature of $25^{\circ \mathrm{C}}$ and cold shock $\left(0^{0 \mathrm{C}}\right)$ at different times $(0.5,2,4,6,8$ minutes $)$. The results showed that the cyanobacteria are able to maintain their survival in cold shock treatments. In cyanobacteria Anabaena shocks cause a lag phase. In cyanobacteria Nostoc, behaviors were different related to thermal shocks and by contrast Anabaena within 6 minutes had negative growth the fifth day. The photoperiod shock eight minutes was not observed a significant difference in the carotenoid content. To achieve stability in the filament part of Phycobilisomes shocks have played a similar role in half and eight minutes. However, the system of will be affected in the short times. It appears that chlorophyll formation in relation to the time and thermal shock in these cyanobacteria varies depending on the species.
\end{abstract}

Key words: Anabaena, Nostoc, habituation, cyanobacteria, thermal shock

\section{INTRODUCTION}

Blue-green algae (BGA), also known as cyanobacteria, are a phylum of bacteria that utilize photosynthesis to obtain energy. BGA have been used as food or medicine by humans in Asian, African and South American countries for centuries (1). Nitrogen-fixing cyanobacteria, the major component of microbial flora in rice paddy fields, are the main contributors to photo dependent $\mathrm{N}_{2}$ fixation (2). Fischerella, as well as all cyanobacteria, utilizes nitrate, nitrite and ammonium as the sole nitrogen source for growth. As cyanobacteria are the early microorganisms on the earth which contribute to altering the atmosphere, they have several adaptive mechanisms to survive in extreme or variable environments (3). Life on earth has evolved in a sea of natural electromagnetic fields (EMFs). Over the past century, this

\footnotetext{
*Correspondence to: $R$. Taheri, Department of Biology, Gorgan Branch, Islamic Azad University, Gorgan,Iran, raziyehtaheri65@gmail.com
}

natural environment has sharply changed with introduction of a vast and growing spectrum of man-made electromagnetic fields (4). Since the 18th century, scientists have been intrigued by the interaction of EMFs and various life processes (4). The cosmopolitan community of cyanobacteria has a significant role in N2 fixing improving the fertility of wetlands such as rice fields (5). They are common inhabitant of aquatic and terrestrial surfaces, including extreme environment (5). Also the ability of producing biologically active compounds makes them a rich source of potentially useful natural products and the targets of screening programs (6). Biological soil crusts occur in semiarid and arid regions throughout the world, and they have been proved to play an important role in maintaining or improving the state of the environment (7). However, due to their sensitivity to anthropogenic and natural disturbances, they are in a degraded state in many areas throughout their range (7). 
It is well documented yet that cyanobacteria have a remarkable flexibility to adapt to a wide range of environmental conditions. Nostoc is a cyanobacteria from Nostocales order that assume a special significance in these environments (8). Species of Fischerella have been reported from North and South of Iran (9). Necessity of acclimation to light, $\mathrm{pH}$ and carbon dioxide concentration fluctuations is an example of these common aspects. Acclimation to light intensity has been the topic of numerous physiological, ultra structural and biochemical studies on a variety of cyanobacteria strains (10). Furthermore, in each of these habitats, widely fluctuating environmental parameters, including light level and quality, temperature and mineral nutrient availability, interact to influence growth, molecular resource allocation, and photosynthesis through complex acclamatory strategies (10). Fischerella show unique potentialities including antibacterial, biotransformation and allelopathic features (9). Albertano et al. (11) have studied the effect of photosynthesis on $\mathrm{pH}$ variation in cyanobacteria biofilms from Roman catacombs made by Scytonema (and a little Fischerella) species using potentiometric micro sensors. Results have shown that both morphological and physiological patterns changes with $\mathrm{pH}$ and irradiance. They have collected cyanobacteria at photosynthetic photon flux density (PPFD) below $2.5 \mu$ mol photon m-2 s1. Until now we have published some papers about taxonomy (molecular and traditional) and Eco physiology (light and $\mathrm{pH}$ acclimation but not combination) (9). Changes in $\mathrm{pH}$ affect the solubility and bioavailability of nutrients (12), transport of substances across the cytoplasmic membranes, and the activity of intra- and extracellular enzymes, as well as photosynthetic electron transport and the osmotic potential of the cytoplasm (13). Many studies have verified that cyanobacteria were able to tolerate a certain degree of drought and salinity, including the accumulation of extracellular substances, the protection of antioxidative system and the adjustment of other metabolic mechanism (14). Pierangelini et al. (15) indicated that $C$. raciborskii NPD responds to low light by changing the size of the light harvesting antenna. Ecologically, the physiological acclimations observed suggest that $C$. raciborskii NPD can perform well in environments where light intensity is low and relatively stable, as might occur during the final stages of HABs (Harmful Algal Blooms) (15). Studies of the acclimation of cyanobacteria usually consider only one environmental factor at a time. However, under natural conditions, cyanobacteria are exposed to the combined influences of several factors. The objective of this study was to examine some physiological characterization of common cyanobacteria Nostoc sp. SF77 and Anabaena sp. FS 76 species of the natural isolate from northern of Iran under thermal shock condition.

\section{MATERIAL AND METHOD}

In most developed countries, banks are there for algae whereas unfortunately, in Iran the possibility of direct access to the native algal genus or species is impossible. So to produce pure colonies of a particular species must personally collect and purify the desired species. Soil samples (the soil surface to a depth of $2.5 \mathrm{~cm}$ ) collected from rice fields of Northern Provinces of Iran (Golestan, Mazandaran and Guilan). In order to culture the soil, some of the soil samples taken from 2 $\mathrm{mm}$ were passed through a sieve and then Medium BGO - 11 were added. After colonization and isolation, the cyanobacteria Nostoc sp. SF77 and Anabaena sp. FS 76 was purified. Various methods have been proposed to separate algae from each other, which could include the method "agar" noted (16) that were used in this study. Due to the difficulty and complexity taxonomy of cyanobacteria, it is required to the experience, education and existence of sufficient resources. There is no source of cyanobacteria in Iran and therefore must rely on the experience. The following keys are valid and in use $(17,18,19)$. A loopful cyanobacterial biomass were inoculated in $250 \mathrm{ml}$ conical flasks and incubated in culture room at $28 \pm 2^{\circ} \mathrm{C}$ of $4 \mathrm{KLux}$ light intensity provided by white fluorescent tubes 14/10 light dark phase. During this period, cultures are not exposed to aeration by gas or agitation. After a period of incubation cultures were treated. The algal suspensions were completely agitated in order to produce homogeneous samples. Samples for half an hour were centrifuged (2500 round per minute). Then the supernatant (culture medium) was discarded. After three times culture medium BGO - $11(5 \mathrm{ml})$ added in order to produce homogeneous again. Finally medium was brought to $20 \mathrm{ml}$ volume. In each treatment, factors were regarded as variable and environmental factors were kept constant. Laboratory flasks daily for one hour were to blink on a shaker flask apparatus. Also they have grown in terms of different temperature shock $(0.5,2,4,6,8)$ by using ice and the turbidity was measured by a spectrophotometer. Estimation of phycobiliproteins was determined by the 
method described by (20). $10 \mathrm{ml}$ algal suspension was centrifuged at $7000 \mathrm{rpm}$ for 10 min (refrigerated centrifuged eppendorf 5430 $\mathrm{R})$. The pellets were suspended in $5 \mathrm{ml}$ phosphate buffer. The contents were repeatedly freeze in $4^{\circ} \mathrm{C}$ and thawed at room temperature. The supernatants were pooled and the absorbance was measured at $562 \mathrm{~nm}, 615 \mathrm{~nm}$ and $652 \mathrm{~nm}$ for phycocyanin, allophycocyanin and phycoerythrin respectively using UVvisible spectrophotometer (Shimadzu 1800). Estimation of total carotenoids was determined by the method described by (21). $10 \mathrm{ml}$ homogenized algal suspension was taken and centrifuged at $6500 \mathrm{rpm}$ for $10 \mathrm{~min}$ (refrigerated centrifuged eppendorf $5430 \mathrm{R}$ ). Discarded the supernatant and added $3 \mathrm{ml} \mathrm{85 \%}$ acetone and subjected to repeat freezing and thawing until the pellet becomes colorless. Measured the volume of the extract and make up the final volume upto $10 \mathrm{ml}$ with $85 \%$ acetone and read the O.D. at $450 \mathrm{~nm}$ using $85 \%$ acetone as blank and calculated the total amount of carotenoids in $\mu \mathrm{g} / \mathrm{ml}$ using UVvisible spectrophotometer (Shimadzu 1800). Estimation of chlorophyll-a was determined by adapting the method described by (22). $10 \mathrm{ml}$ of homogenized algal suspension was taken in centrifuge tube and done centrifugation at 7000 $\mathrm{rpm}$ for $10 \mathrm{~min}$ and then discarded the supernatant and transferred the algal pellet to a test tube and added $10 \mathrm{ml}$ of $90 \%$ methanol. Shaked the contents and placed the tubes covered with aluminium foil in a water bath at $60^{\circ} \mathrm{C}$ for $30 \mathrm{~min}$. The absorbance from supernatant was measured at $665 \mathrm{~nm}$ against methanol blank (UV-visible spectrophotometer (Shimadzu 1800). Tests that have several main components, correlation, analysis of variance without replication and other 2-factor statistical analysis were analyzed using the software SPSS ver 16, Sigma plot ver 8 and Past.

\section{RESULTS AND DISCUSSION}

In Anabaena sp. FS 76, thermal shock (0, 5, 2 and 4 minutes) not only creates lag phase but also the entrance of negative growth (Figure 1). Thermal shock is palpable impact on growth curves and for interrupting the reproduction of the first few days after the inoculation. Although the sample can pass negative growth and the exponential growth of import. Temperature shock for eight minutes, not only caused negative growth, but it causes the exponential growth phase from the first day due to cyanobacteria habituation to the new condition in this time. In a study, the results showed that in cyanobacteria, species or sample with more droughts tolerant, have more tolerance freezing. Indeed, in examples like Nostoc sp. commune life in the community form (colonies) result in the combined resistance against drought and freezing the (23). It seems that the habituation behavior of the two species is different to thermal shock and with this similarity that in both very shortterm thermal shock (30 Seconds) makes higher growth rate (Figure 1, 2). In natural conditions, soil habitats, limiting factors such as carbon dioxide and light restrictions, with both the sample and cyanobacteria should be compatible with the conditions set (24). Thus, the combination of more realistic information about the mechanism of habituation cyanobacteria provides (24). Generally, there have been almost entirely similarities and differences compared to studies that show particular physiological of the three cyanobacteria (Fischerella - Nostoc Anabaena) with two samples Microchaete sp. Fs 13 and Nostoc sp. FS 101, under the combined effects of limited light and carbon dioxide and salt (different concentrations of sodium chloride) and acidity and alkalinity (11). However, there will also be many differences both in growth and in the photosynthetic pigment and the performance $(10,11)$. As shown in Figure 3, terms of time causes significant changes in the phycocyanin content of Anabaena sp. FS 76. Thermal shock four minutes causes the phycocyanin content on the fourth day to be increased significantly. This is the fifth and eighth days after inoculation associated with a significant decrease. Although in Nostoc sp. SF77 it is similarity with Anabaena sp. FS 76 significant increase in phycocyanin on the fourth day, but differences caused by the different impact of thermal shock at the time more clearly show species-dependent behavior (Figure 4). Phycocyanin content in Anabaena sp. FS 76 in the long term (more than eight days after inoculation) significantly affected by thermal shock is reduced more than two minutes while the Nostoc sp. SF77, it is not significant, although naturally it can be seen some differences. According to phycocyanin content in the eight minute treatment in both cyanobacteria especially in the eighth day, collection systems light for photosynthesis, during the eight minute treatments over time the extent to arrive that they are consistent with habituation. The accuracy of the growth curves show that the growth in both, the eight minutes treatment makes greatest growth (Nostoc sp. SF77) and most habituation (Anabaena sp. FS 76). So, the eight minute treatment creates a situation somewhat similar to natural conditions, but time has a significant impact on 
this issue. The eight minute treatment can be considered as an appropriate time for habituation and thus it is somewhat similar to a reproductive cycle. Phycocyanin is the major and important phycobiliproteins which exhibited anticancer activity, stimulation of immune system and ability to treat ulcers and haemmorrhoidal bleeding. Since the culture conditions are known to be changed the biochemical composition of the algae the constituents can be improved upon further by manipulating culture conditions (24).

\section{Growth Curve Dry weight Anabaena sp. FS}

76/temperature shock.Comparison

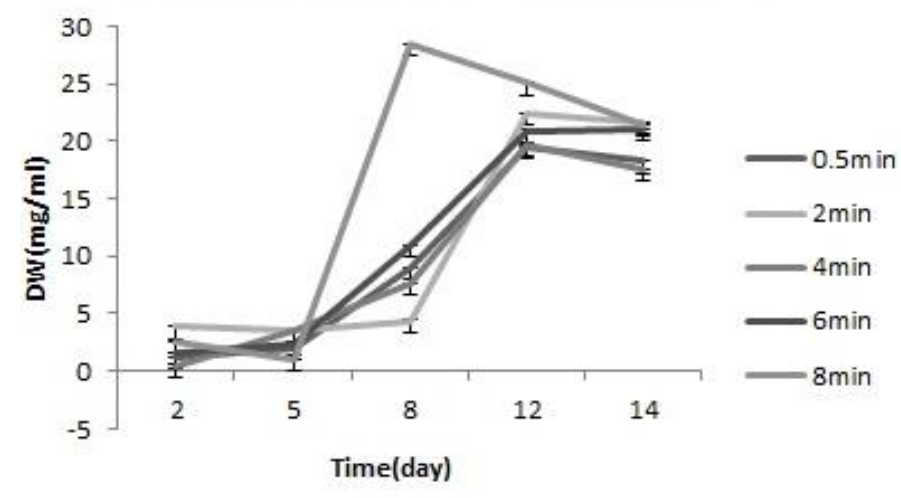

Figure 1. Growth curve dry weight Anabaena sp. FS 76 under different temperature shock.

\section{Growth Curve Dry weight Nostoc sp.FS77 / temperature shock.Comparison}

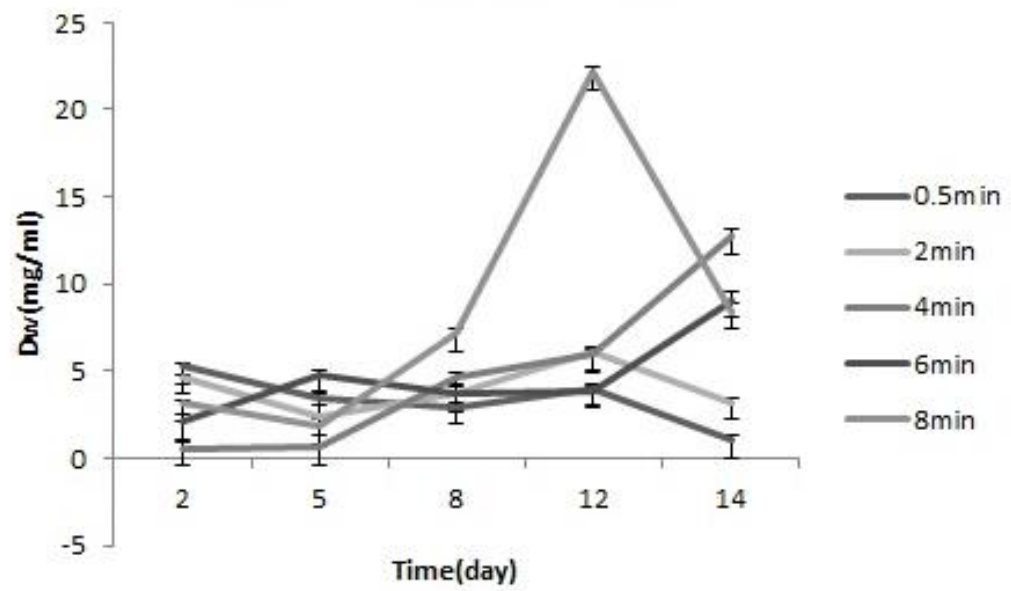

Figure 2. Growth curve dry weight Nostoc sp. SF77 under different temperature shock.

Phycocyanin / Anabaena sp. FS 76

/temperature shock.Comparison

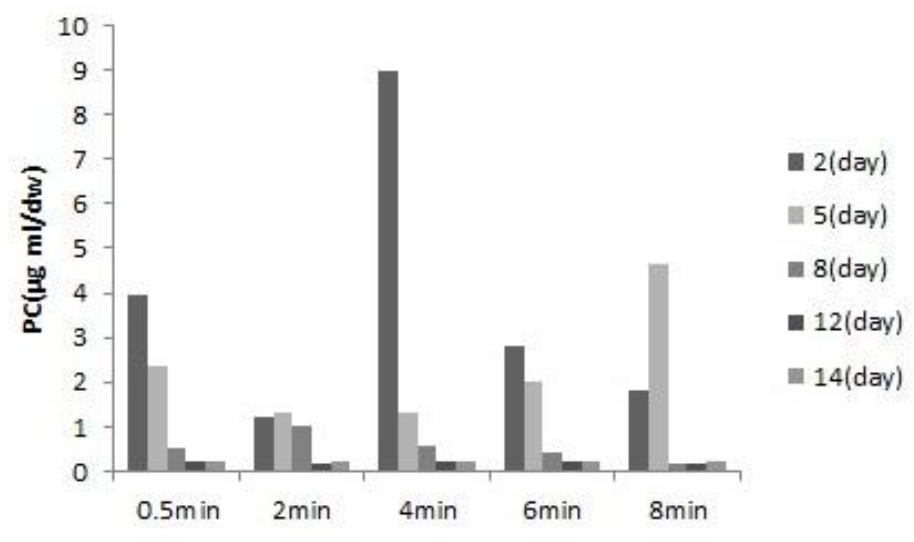

Figure 3. Phycocyanin content in Anabaena sp. FS 76 under different temperature shock. 


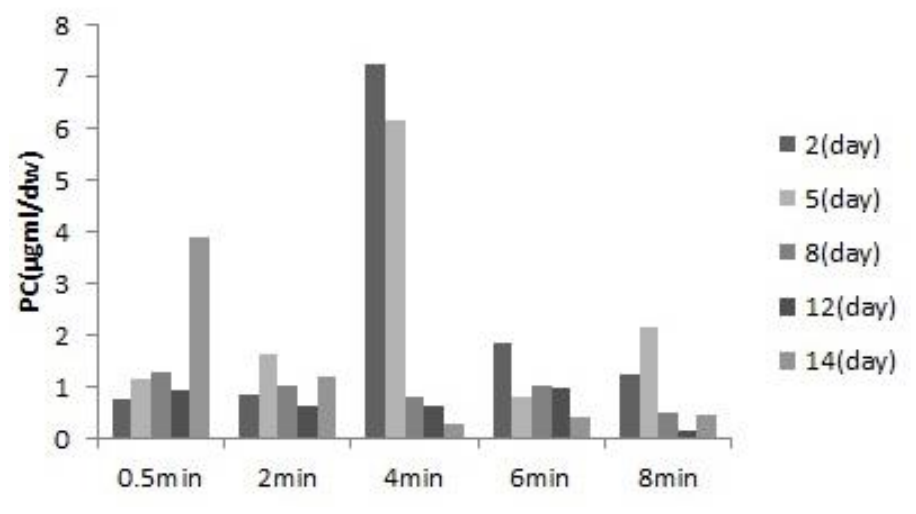

Figure 4. Phycocyanin content in Nostoc sp. SF77 under different temperature shock.

The accuracy of the content of carotenoids Nostoc sample (Figure 5) shows the optical shock treatment eight minutes, carotenoids are stable and no significant difference between the content of carotenoids is observed on different days. By examining the levels of carotenoids Anabaena can see that content on different days of relative stability (Figure 6). It can be concluded that these two carotenoids in cyanobacteria are stable under thermal shocks and are not affected by eight minute's thermal shocks. The content of phycoerythrin both cyanobacteria are shown in (Figure 7, 8). The comparison shows that the eight minute's thermal shocks, phycoerythrins show stability from the eighth day onwards such as phycocyanin and carotenoids. It could be further evidence in stability of phycobilisome and light collection system for eight minute light treatments. Another interesting point there is no difference between 30 seconds and eight minutes thermal shocks. It seems that a combination of limited light intensity, time and thermal shocks change allophycocyan in both cyanobacteria (Figure 9, 10). In the cyanobacteria Anabaena sp. FS 76 (Figure 9), thermal shocks over time (eight days later), decrease the amount of allophycocyanin be the same. In other words, the influence of different time's thermal shock during these days, there is no significant difference. It seems that cyanobacteria in the central part of phycobiliproteins enlarge with time, loses its sensitivity to thermal shocks (23). Nostoc cyanobacteria behavior in this regard varies for different time and temperature shocks affect the production of allophycocyanin (Figure 10). So only anabaena serves similar phycoeritrin and half and eight minutes on the production of phycobilisome in the central part of no significant impact (23). Short -term (half a minute) and longer term shocks (eight minutes) after the eighth day leads to the meaningless differences in concentrations of chlorophyll and uniformity in production of photosynthesis central part (Figure 11). In Anabaena sp. FS 76, the time and thermal shock act simultaneously and this cyanobacteria obtains the same chlorophyll content. Times less makes significant differences in chlorophyll content. In Nostoc sp. SF77the behavior is somewhat different (Figure 12).

\section{Carotoid / Nostoc sp.FS 77/temperature shock}

.Comparison

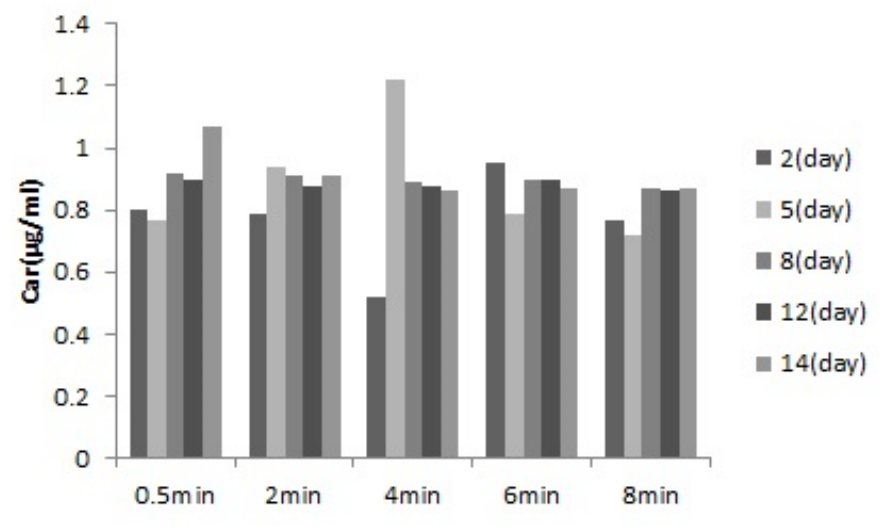

Figure 5. Carotenoids content in Nostoc sp. SF77 under different temperature shock. 


\section{Carotoid /Anabaena sp. FS 76 \\ /temperature shock.Comparison}

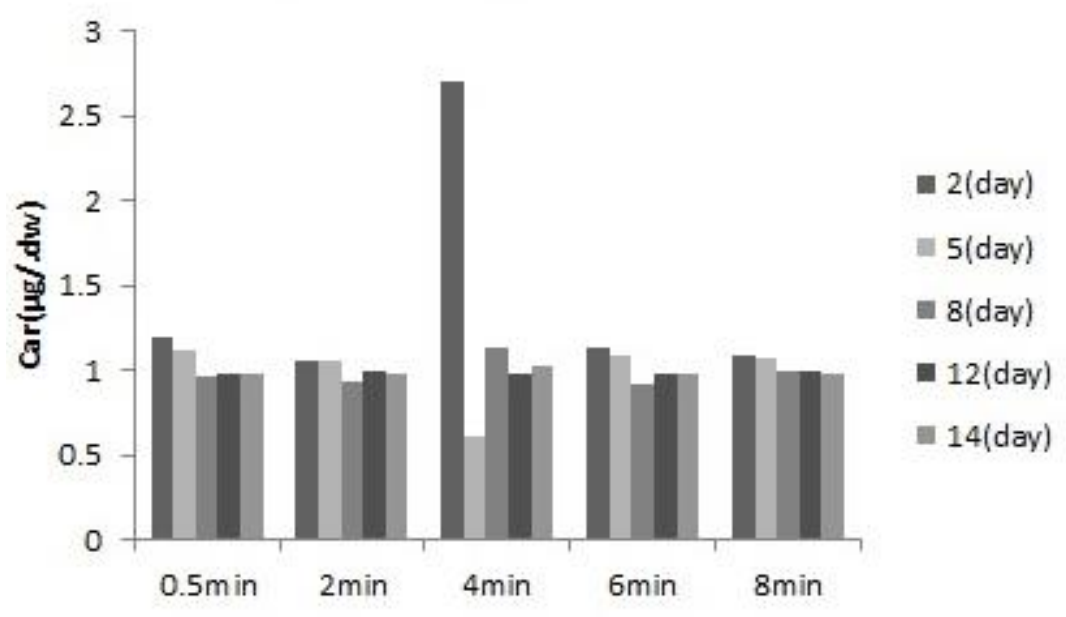

Figure 6. Carotenoids content in Anabaena sp. FS 76 under different temperature shock.

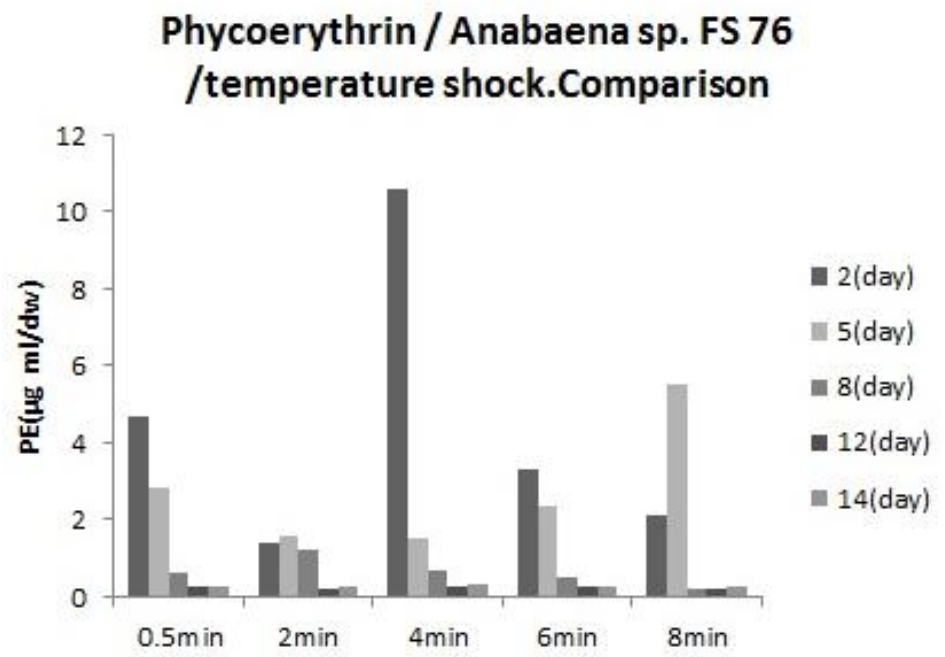

Figure 7. Phycoerythrin content in Anabaena sp. FS 76 under different temperature shock.

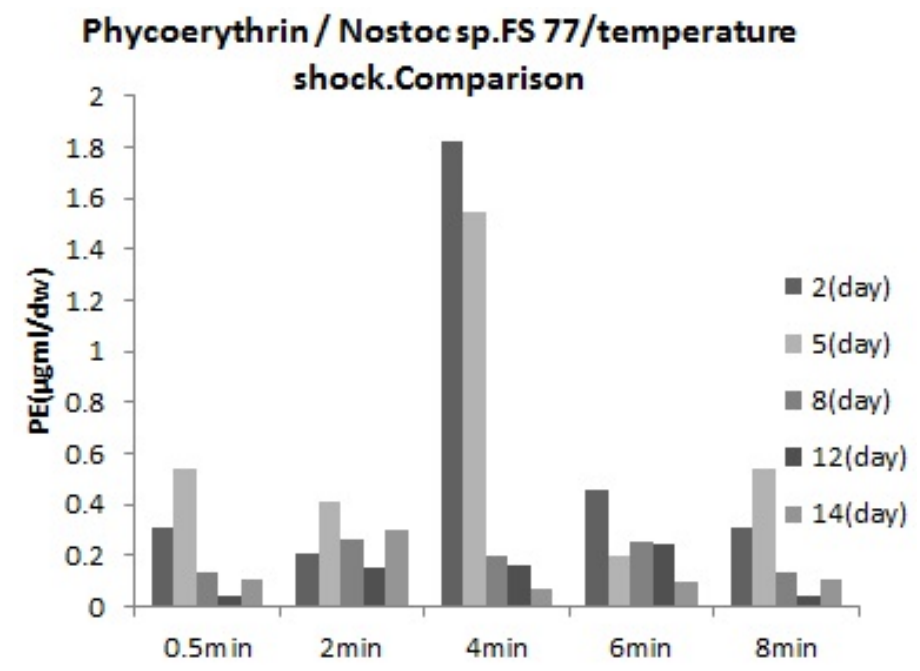

Figure 8. Phycoerythrin content in Nostoc sp. SF77 under different temperature shock. 
Allophycocyanin / Anabaena sp. FS 76

/temperature shock.Comparison

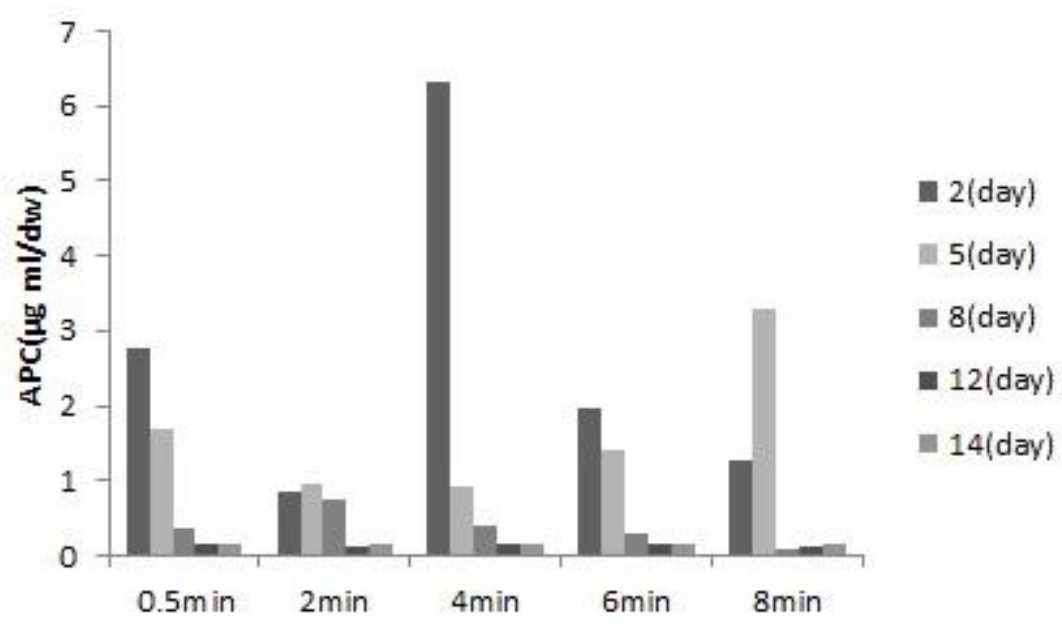

Figure 9. Allophycocyanin content in Anabaena sp. FS 76 under different temperature shock.

\section{Allophycocyanin / Nostoc sp.FS 77 /temperature shock.Comparison}

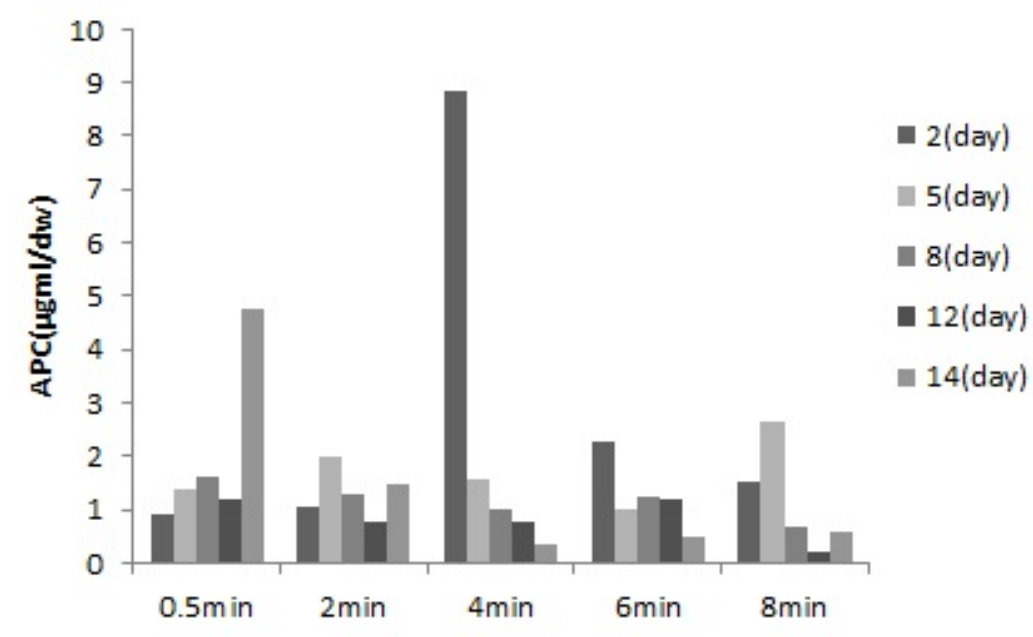

Figure 10. Allophycocyanin content in Nostoc sp. SF77 under different temperature shock.

Chlorophyll / Anabaena sp. FS 76 /temperature shock.Comparison

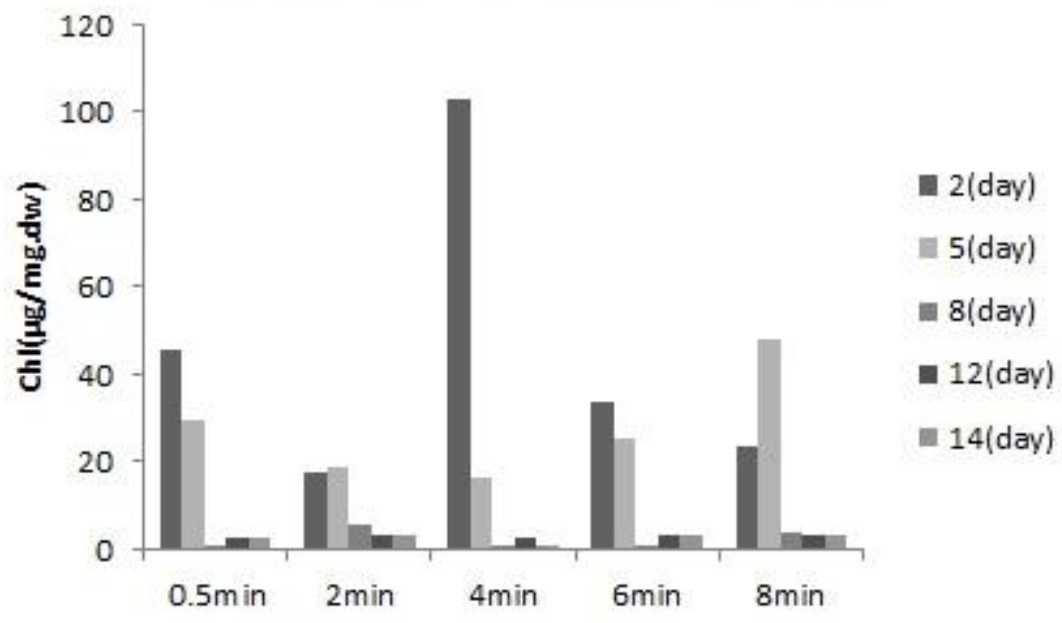

Figure 11. Chlorophyll content in Anabaena sp. FS 76 under different temperature shock. 


\section{Chlorophyll / Nostoc sp.FS 77/temperature \\ shock.Comparison}

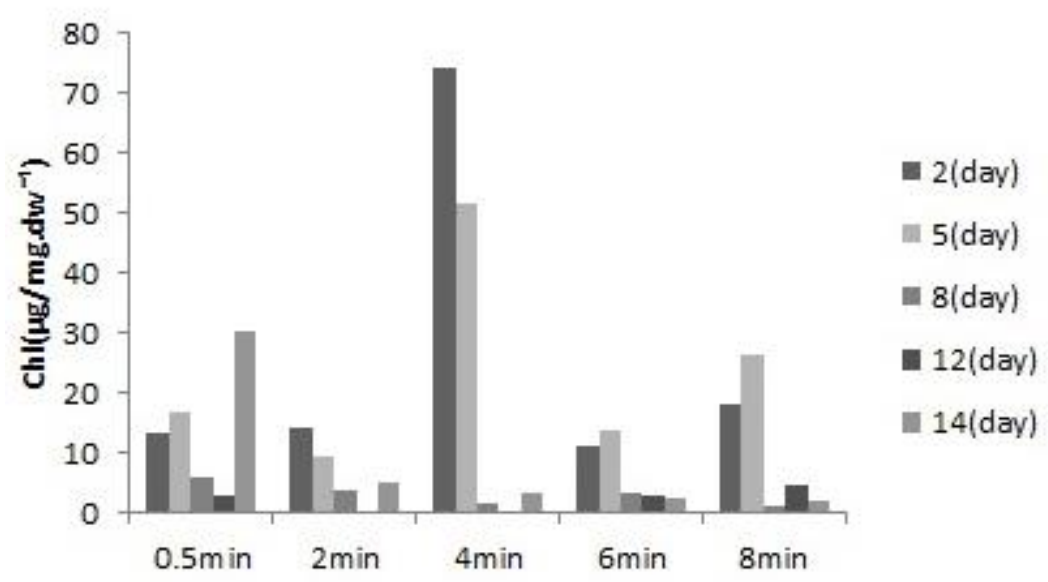

Figure 12. Chlorophyll content in Nostoc sp. SF77 under different temperature shock.

\section{CONCLUSIONS}

It seems that the habituation behavior of the two species is different to thermal shock, in cyanobacteria Anabaena shocks cause a lag phase. In cyanobacteria Nostoc, behaviors were different related to thermal shocks. The eight minute treatment can be considered as an appropriate time for habituation and thus it is somewhat similar to a reproductive cycle.

\section{REFERENCES}

1. Yang, Y., Park, Y., Cassada, D. A., Snow, D.D., Rogers, D. G. and Lee, J. In vitro and in vivo safety assessment of edible blue-green algae, Nostoc commune var. sphaeroides Kützing and Spirulina plantensis. Food Chem. Toxicol, 49(7): 1560-1564, 2011.

2. Roger, P.A. and Kulasooriya, S.A. Blue-green Algae and Rice. The international rice research institute, Manila, 112 p, 1980.

3. Tandeau de Marsac, N. and Houmard, J. Adaptation of cyanobacteria to environmental stimuli: new steps towards molecular mechanisms. FMS Microbiol. Rev, 104: 119-190, 1993.

4. Asadi, A., Khavari-Nejad, R.A., Soltan, N., Najafi, F. and MolaieRad, A. Physiological variability in Cyanobacterium phormidium sp. Kützing ISC31 (Oscillatoriales) as response to varied microwave intensities. J. Agri. Tech. 7(3): 665677, 2011.

5. Anand, N., Radha, L., Shanthakumar, R., Hopper,S., Revathi, G. and Subramanian, T.D. Blue-green algaeas biofertilizers: certain view points on the choice of suitable isolates. In Perspective in phycology, ed. Rajarao, V.N. pp. 383-391, 1990.

6. Soltani, N., Khavari-Nejad, R.A., Tabatabaei Yazdi, M., Shokravi, SH., Growth and Some Metabolic Features of Cyanobacterium Fischerella Sp. FS18 in Different Combined Nitrogen Sources. J. Sci. Islamic Republic of Iran, 18(2): 123-128, 2007.

7. Wang, W, Liu, Y., Li, D., Hu, C., Rao, B Feasibility of cyanobacterial inoculation for biological soil crusts formation in desert area. Soil biology and biochemistry, 41: 926-929, 2009.

8. Iranshahi, S., Nejadsattari, T., Soltani, N., Shokravi, S., Dezfulian, M. The effect of salinity on morphological and molecular characters and physiological responses of Nostoc sp. ISC 101. Iranian Journal of Fisheries Sciences, 13(4): 907-917, 2014.

9. Soltani, N., Siahbalaie, R. and Shokravi, SH. Taxonomical characterization of Fischerella sp. FS18- A multidisciplinary approach, Inter. J. Algae, 1:48 -55, 2011.

10.Safaei Katoli, M., Nejadsattari, T., Majd, A., Shokravi, S. Physiological, morphological and ultrastructural to combination responses of Cyanobacterium Fischerella sp. FS 18 effects of extreme conditions. J. Appl. Environ. Biol. Sci, 5(1):135-149. 2015.

11.Albertano, P., Bruno, L., D'Ottavi, D., Moscone, D. and Palleschi, G. Effect of photosynthesis on $\mathrm{pH}$ variation in cyanobacteria biofilms from Roman 
catacombs. J. Applied Phycol, 12: 379$384,2000$.

12.Stumn, W., Morgan, J.J. Aquatic Chemistry. 2nd ed. Wiley, NewYork, 1981.

13.Poza-Carrion, C., Fernandez-Valiente, E., Pinas, F. F. and Leganes, F., Acclimation of photosynthetic pigments and photosynthesis of the cyanobacterium Nostoc sp. Strain UAM 206 to combined flactuations of irradiance, $\mathrm{pH}$, and inorganic carbon availability, J. Plant Physiol, 158: 1455- 1461, 2001.

14.Lan, S., Wu, L., Zhang, D., Hu, C., Liu, Y. Effects of drought and salt stresses on man-made cyanobacterial crusts. Euro. J. Soil. Biol, 46:381-386, 2010.

15.Pierangelini, M., Stojkovic, S., Orr, P., Beardall, J. Photo-acclimation to low light - Changes from growth to antenna size in the cyanobacterium Cylindrospermopsis raciborskii. Harmful Algae, 46: 11-17, 2015.

16.Belcher, H., Swale, E. Culturing algae, a guide for schools and colleges, Tilus Wilson and Son LTD, 1982.

17. Castenholz, R. W. Phylum BX. Cyanobacteria. In Bergey's Manual of Systematic Bacteriology, 2nd edn. Edited by D. R. Boone \& R. W.
Castenholz. New York: Springer, pp. 473-487, 2001.

18.Prescott, G.W. Algae of the Western Great Lakes Area. $2^{\text {nd }}$ ed. Wm. C. Brown Co., Dubuque, Iowa p. 977, 1962.

19.Desikachary, T.V. Cyanophyta, Indian Council of Agricultural Research, New Delhi. p.686. 1959.

20.Bennett, A., Bogorad, L. Complementary chromatic adaptation in filamentous blue- green algae, $J$. Cell Biol, 58: 419-433,1973.

21.Jensen, A. Chlorophylls and carotenoids, In J. A. Hellebust and I. S. Craige, (eds.), Handbook of phycological methods: Physiological and biochemical methods, Cambridge University press, pp. 59-70. 1978.

22. Mckinney, G. Absorption of light by chlorophyll solution, J. Biol. Chem, 140: 315-322. 1941.

23.Acea, M. J., Prieto-Fernandez, A., Diz-Cid, N. Cyanobacteria inoculation of heated soils: effect of microorganisms of $\mathrm{C}$ and $\mathrm{N}$ cycle on chemical composition in soil surface. Soils boil. Biochem, 35: 513 - 524, 2002.

24.Ciferri, O. Spirulina: The edible microorganism, Microbiol. Rev, 47(4): $551,1983$. 